\title{
Le Marché Dans La Construction De L'inter- Territorialité Transfrontalière : Le Cas Du Marché De Kye-Ossi Dans L'analyse A Travers La Distribution Des Produits Agricoles Entre Le Cameroun, La Guinée Équatoriale Et Le Gabon (Sud Cameroun)
}

\author{
Hervé Tchekote, \\ Frankie Herman Tchinda, \\ Chapgang Noubactep, \\ Mérimé Aguekam Ngako, \\ Département de Géographie, Université de Dschang, Cameroon
}

Doi:10.19044/esj.2020.v16n11p205 URL:http://dx.doi.org/10.19044/esj.2020.v16n11p205

\section{Résumé}

Depuis plus de trois décennies, le marché de Kyé-Ossi s'est positionné comme une place centrale dans les échanges Sous-régionales des produits agricoles entre le Cameroun pays producteur, le Gabon et de la Guinée Équatoriale pays consommateurs au regard de sa position frontalière. Analysant cette dynamique, le présent travail s'est proposé de montrer le rôle de ce marché dans l'approvisionnement et dans la construction de l'interterritorialité transfrontalière au Sud du Cameroun. Sur la base d'observations, d'entretiens semi-structurés auprès des personnes ressources et des questionnaires auprès d'une centaine d'acteurs diversifiés dont les commerçants (35), les transporteurs (20) et les acheteurs étrangers (45), l'étude montre comment un important flux de produits agricoles se construit entre l'arrière-pays (Cameroun) et le marché de Kyé-Ossi, avec comme objectif alimenter un réseau d'approvisionnement des villes Gabonaises et Équato-guinéennes entre autres. Les acteurs qui interviennent dans ce marché se spécialisent en construisant des circuits de commercialisation inter-états. Le marché de Kyé-Ossi contribue ainsi à l'intensification des échanges et des mobilités économiques entre le Cameroun, le Gabon et la Guinée Équatoriale. Cependant, ces échanges quoique fructueux se heurtent à la non effectivité de la libre circulation des personnes au niveau de la sous-région, qui est pourtant un acquis des politiques publiques inter-états.

Mots clés: Marché, hinterland, approvisionnement, produits vivriers, KyéOssi, Cameroun, Gabon, Guinée Équatoriale 


\title{
The Kye-Ossi Market (South Cameroon) in the Construction of Cross-Border Inter-Territoriality: Analysis Through the Distribution of Agricultural Products Between Cameroon, Equatorial Guinea and Gabon
}

\author{
Hervé Tchekote, \\ Frankie Herman Tchinda, \\ Chapgang Noubactep, \\ Mérimé Aguekam Ngako, \\ Département de Géographie, Université de Dschang, Cameroon
}

\begin{abstract}
For more than three decades, the Kye-Ossi market has positioned itself as a central place in sub-regional exchanges of agricultural products between Cameroon producing country, Gabon and Equatorial Guinee consuming countries with regard to its border position. Analysing this dynamics, the present work proposes to question the role of this market in the construction of cross-border inter-territoriality in south Cameroon. On the base of observations, semi-structured interviews with a hundred of diversified actors amongst which traders (35), transporters/carriers (20) and (45) foreign buyers. The study shows how an important flow of agricultural products is built between the hinterland of Cameroon and the Kye-Ossi market, with objectif of suppling a supply network to the Gabonese and Equatorial Guinea towns amongst others. Actors that intervene in this market are specialised in the building of interstate commercial circuits. The Kye-Ossi market contributes as such to the intensification of exchanges and economic mobilities in Cameroon, Gabon and Equatorial Guinea. These exchanges although fruitful, come against the ineffectiveness of the free movement of an asset of interstate public policies.
\end{abstract}

Keywords: Markets, hinterland, supply, food products, Kye Ossi, Gabon, Equatorial Guinea 


\section{Introduction}

$\mathrm{Au}$ lendemain des indépendances, les États africains se sont investis dans le chantier de la construction des grands ensembles politiques et économiques régionaux et sous régionaux avec comme leitmotiv une meilleure intégration des populations, la suppression des frontières et l'intensification des échanges intra-régionaux (Dieye, 2006, p.1-2). Si de telles initiatives ont connu des résultats plus ou moins appréciables en Afrique de l'Ouest et du Sud, elles demeurent très mitigées en Afrique Centrale où la volonté des États contraste avec les réalités du terrain. La persistance des contrôles aux frontières du Gabon et de la Guinée Équatoriale, l'expulsion des Camerounais de la Guinée Équatoriale vers l'île de Campo au Cameroun, la fermeture unilatérale des frontières Équato-guinéennes sont autant de mesures qui donnent plus d'allants aux initiatives individuelles que collectives des États en matière d'aménagement des territoires transfrontaliers. Ainsi, au Cameroun le Document de Stratégie pour la Croissance et l'Emploi (DSCE) réaffirme la volonté de l'État à développer et à diversifier ses échanges commerciaux au plan national et international. Dans cette perspective, le Cameroun entend assumer sans ambages un rôle de leadership dans la Sousrégion à travers la création et l'extension des marchés transfrontaliers considérés comme un indicateur fiable de la performance économique (DSCE, 2009 :16). Généralement crées en marge des décisions municipales ou par les mairies dans le cadre de la planification urbaine, ces marchés sont perçus comme des lieux d'échange des biens et services, mieux encore comme des leviers de développement et d'intégration économique (Degavre et Lemaître, 2008, p 3-6 ; Ihle et al., 2010, p41-42, Mabaya Edward, 2004, p33).

Le marché de Kyé-Ossi rentre dans cette dynamique. Créé spontanément par des populations soucieuses de s'extirper du cercle vicieux de la pauvreté, puis toléré, maintenu, reconnu et restructuré par les autorités municipales, ce marché assure l'approvisionnement des populations locales et de la Sous-région en produits vivriers. En moins de trois décennies, il est devenu un véritable espace d'échanges entre le Cameroun, le Gabon et la Guinée Équatoriale, malgré la fermeture constante des frontières de part et d'autre. Dans ce contexte contrasté, entre les politiques publiques qui prônent le libre-échange entre les pays de la Sous-région et la fermeture quasi constante des frontières par certains pays voisins, l'objectif de ce travail est de montrer le rôle de ce marché dans l'approvisionnement et dans la construction de l'inter-territorialité transfrontalière au Sud du Cameroun. Dès lors, quelle est donc la place qu'occupe ledit marché dans l'approvisionnement des populations des pays frontaliers au Sud du Cameroun ? 


\section{Outils et méthodes}

\subsection{Présentation de la zone d'étude}

Kyé-Ossi, Chef-lieu de la commune du même nom, est situé dans le département de la vallée du Ntem dans le Sud du Cameroun ; précisément entre le $210^{\prime} 31^{\prime \prime}$ Nord et le $11^{\circ} 20^{\prime} 27^{\prime \prime}$ 'Est (figure 1).

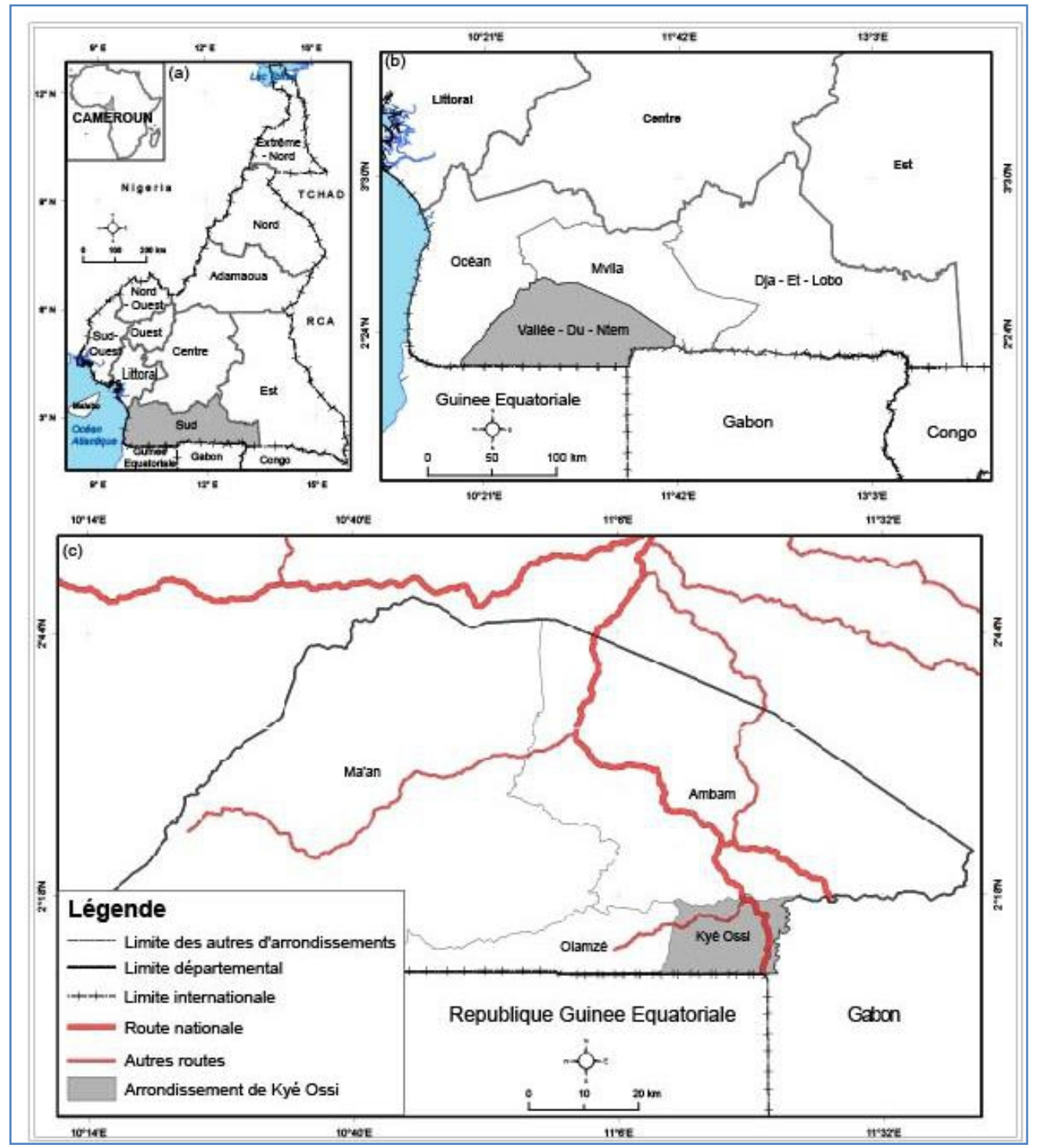

Figure 1 : Localisation de la zone d'étude.

(Source : Carte administrative du Cameroun ; Conception et réalisation : Tchinda, 2018)

Il est limité au Nord par la localité d'Akombang (Cameroun), au Sud par le village Meyoke (Gabon), à l'Ouest par la ville d'Ebebeyin (Guinée équatoriale) et à l'Est par le village Mefoup (Cameroun). Rarement évoquée, 
la localité ne figure pas sur les cartes nationales représentant le Cameroun au $1 / 500000^{\mathrm{e}}$. Seule la couverture régionale du pays au $1 / 200000^{\mathrm{e}}$ signale sur la feuille d'Ebolawa, ce « village-rue » à la situation géographique peu banale. En effet, Kyé-Ossi est implanté au carrefour de trois États, l'une des rues du village aboutissant au Gabon, l'autre en Guinée Équatoriale. Le village se trouve ainsi cerné par des postes frontières. S'il est éloigné d'Ambam au Cameroun d'une trentaine de kilomètres et de Bitam au Gabon de 25 kilomètres, le marché n'est qu'à 03 kilomètres d'Ebebeyin en Guinée Équatoriale.

Ce marché dit frontalier de Kyé-Ossi s’est développé dans la deuxième moitié des années 70 à la faveur de l'ouverture des frontières Équatoguinéennes survenue suite au changement de régime. L'entrée de la Guinée Équatoriale dans la zone Franc en 1985 renforce l'animation de Kyé-Ossi et amène maints commerçants exerçant jusqu'à alors à Ambam, à se délocaliser vers cette localité frontalière pour se rapprocher de la clientèle Équatoguinéenne. Depuis la fin des années 1993, il est en plein essor suite au déclin du marché d'Abang Minko'o.

\subsection{Collecte et analyse des données}

Cette étude a exploité deux types de données.

Les données secondaires, issues des mémoires, des ouvrages, et des articles scientifiques traitant de l'approvisionnement vivier et de l'interterritorialité frontalière. Ces travaux ont été repérés au département de géographie de l'Université de Dschang, au département de géographie de l'École Normale de Bambili, à la bibliothèque centrale de l'Université de Dschang, ainsi que sur internet.

- Les données primaires collectées à travers l'observation directe, les enquêtes semi-structurées et les enquêtes par questionnaire. Les entretiens ont été conduits auprès des personnes ressources, dont les autorités administratives, municipales, traditionnelles et des leaders d'association. Les questionnaires pour leur part ont été adressés aux commerçants (35), transporteurs (20) et aux clients (45) qui constituent les principaux agents de l'échange. L'échantillon s'est constitué par choix raisonné et a permis d'aller au contact des enquêtés.

Les données recueillies quant à elles ont été analysées à l'aide du logiciel Excel. Les cartes ont été traitées par le logiciel Argis.

\section{Résultats}

\section{Un marché dynamique aux infrastructures très sommaires}

Le marché frontalier de Kyé-Ossi est un marché quotidien. Cependant, les pics d'activités ne sont enregistrés que trois fois par semaine, notamment les lundis, mercredis et vendredis. Ces jours correspondent à l' 'érrivée des 
Gabonais et des Équato-guinéens en quête d'approvisionner sur ce marché. (Ayiwoue Mbouemboue et al., 2006, p24). Il représente un principal point à partir duquel le Gabon et la Guinée Équatoriale s'approvisionnent en produits agricoles. Les Camerounais s'y approvisionnent en vins, en liqueurs d'Espagne et en produits cosmétiques. Le marché couvre une superficie d'environ $20000 \mathrm{~m}^{2}$ pour une capacité d'accueil en 2013 de près de 2500 places (PCD, 2014). Ses bâtiments, constitués essentiellement de magasins et des hangars s'organisent autour de deux grands espaces commerciaux :

- Le premier, plus ancien, a été aménagé autour des années 2005 par des particuliers. Il est réservé au commerce des produits agroalimentaires, électroménagers et manufacturés.

- Le deuxième espace commercial pour sa part est spécialisé dans la commercialisation des produits vivriers, dont certains tels que le maïs, le haricot, la pomme de terre et la tomate font l'objet de commerce régional (Ayiwoue Mbouemboue et al., 2006, p24). Il a été aménagé en 2005 par la mairie sous financement du Programme National de Développement Participatif (PNDP) dans l'optique de décongestionner le marché.

Bien que doté d'un hangar d'une capacité d'au moins 200 places, le marché de Kyé-Ossi connait une florescence des activités commerciales à ciel ouvert où l'installation des marchandises à même le sol, sur les étals ou sur les comptoirs de fortune sont de mise et présage un déficit dans l'organisation ou la structuration des activités sur ce marché (planche 1). 
Planche 1: Marchandises disposées à même le sol et sur un comptoir de fortune

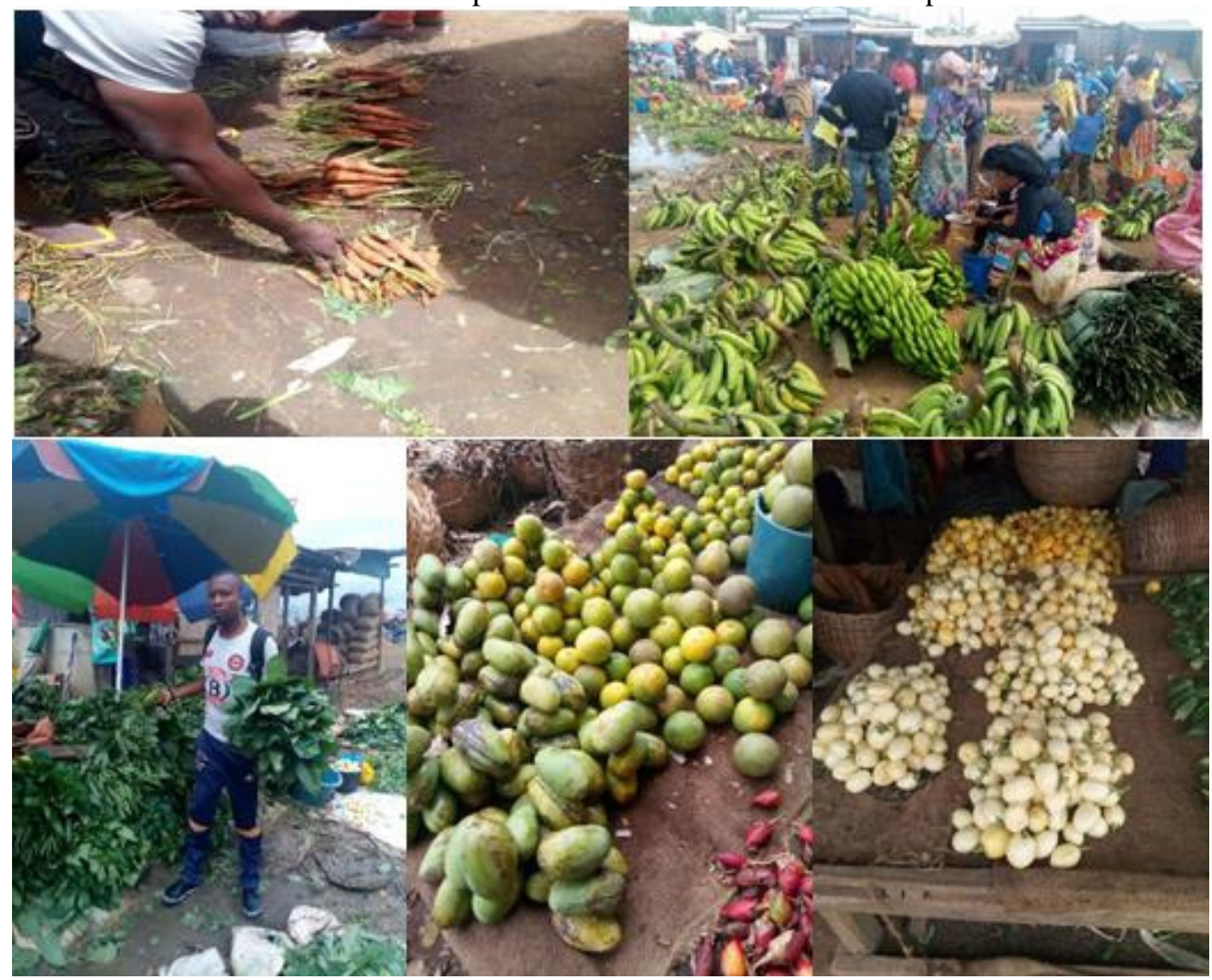

(Source : Image d'enquête, 2018)

Deux raisons justifient la colonisation de ce type de cadre essentiellement par les commerçants du secteur des produits vivriers, des fruits et légumes (Planche 1). D'une part, les coûts locatifs de ces types d'espaces, relativement bas, qui se limitent très souvent au payement de ce qu'il convient d'appeler «ticket du marché », une espèce de taxe communale payée par les commerçants (7500frs/trimestre pour ceux qui ont des comptoirs et $200 \mathrm{frs}$ les jours du marché par tous commerçants). D'autre part, il s'agit de coloniser les artères d'entrée, de sortie et les servitudes du marché afin de se rapprocher de leur clientèle. Certains commerçants disposant des hangars multiplient ces types d'extension à travers le marché.

\section{Des agents de l'échange spécialisés selon la nationalité}

Le marché de Kyé-Ossi met en synergie une pléthore d'agents économiques spécialisés dans les échanges parfois en fonction des nationalités. Ce marché met en synergie une multitude d'agents économiques dont l'engagement sur le marché et la position dans la relation d'échange se 
calquent aisément sur la nationalité. Il s’agit notamment des vendeurs, des acheteurs et des transporteurs.

Les vendeurs, principalement de nationalité camerounaise obéissent à deux systèmes d'approvisionnement: l'approvisionnement en gros et en détails. Encore appelé « bayam sellam » ${ }^{46}$, ils sillonnent les marchés à travers le Cameroun pour acheter auprès des producteurs pour ensuite revendre sur le marché de Kyé-Ossi aux acheteurs Gabonais et Équato-guinéens, comme l'avait déjà relevé Medjou et al., (2007, p.10). Ces «bayam sellam » sont de plusieurs catégories :

- Les grossistes, qui achètent en grande quantité dans les marchés et chez les producteurs des localités lointaines en utilisant comme moyen de transport les camions ;

- Les semi-grossistes qui s'approvisionnent essentiellement chez les grossistes ;

- Les détaillants, qui vendent directement aux consommateurs.

Au cours des dernières décennies, il s'est opéré des mutations dans le système d'approvisionnement du marché, avec les acheteurs gabonais qui passaient outre les «bayem sellam» pour sillonner les campagnes et s'approvisionner directement auprès des producteurs. Cette présence d'acheteurs étrangers dans l'arrière-pays a généré des conflits avec les grossistes sur le territoire camerounais. Les commerçants étrangers étaient en effet à l'origine de la pénurie de certaines denrées alimentaires sur les marchés locaux, puisqu'ils achetaient directement dans les exploitations, sans que les produits ne passent par les marchés locaux où s'approvisionnent entre autres les populations camerounaises. Ce qui a suscité l'intervention des autorités administratives camerounaises qui ont pris des mesures interdisant aux Gabonais d'aller acheter directement auprès des producteurs (Ayiwoue Mbouemboue et al., 2006, p.24). Avec cette intervention des autorités, le marché de Kyé-Ossi, reste le point d'ancrage des échanges entre le Cameroun, le Gabon et la Guinée Équatoriale.

En ce qui concerne les acheteurs, ils sont principalement de nationalité Gabonaise et Équato-guinéenne. Les Gabonais sont organisés en groupes d'une vingtaine de personnes environ. Ils sont spécialisés dans l'achat du plantain et des tubercules. Deux fois par semaine, ils investissent pour acheter les marchandises et mettre dans les camions et de camionnettes en direction du Gabon. Ils arrivent généralement les mardis soirs et les vendredis et mènent leurs opérations commerciales les mercredis et samedis. Les Équato-guinéens pour leur part mènent des actions solitaires.

${ }^{46}$ De l'anglais «buy», acheter et «sell», vendre, le terme «bayam sellam» découle du pidgin (mélange d'un anglais plus ou moins altéré et les langues locales), et désigne littéralement tous ceux qui investissent dans la distribution de gros des vivres. 
Les transporteurs pour leur part sont de diverses nationalités (Camerounais, Maliens, Tchadiens, etc.). Maillons très importants du fonctionnement d'un marché, ils assurent l'acheminement et la distribution des produits des bassins de production vers le marché de Kyé-Ossi et vers les pays voisins. Selon la quantité des produits achetés, les engins utilisés pour le transport vont des camions aux moto-taxi en passant par les camionnettes et les «Opep» ou «clandos ${ }^{47}$. Ces transporteurs sont les maillons essentiels dans la dynamique d'interrelations entre l'hinterland et le marché de KyeOssi.

\section{Le marché de Kye-ossi dans la polarisation de l'hinterland camerounais}

Le marché de Kyé-Ossi est une plateforme incontournable de l'exportation des produits agricoles vers les pays frontaliers comme le Gabon et la Guinée Équatoriale. Les produits qui y sont commercialisés viennent de tout le Cameroun, du nord au sud. Chaque produit arrive sur le marché de KyéOssi à travers un circuit qui lui est spécifique, du bassin de production dans l'hinterland à la frontière du Gabon et de la Guinée Équatoriale. La tomate, les carottes, le piment, le maïs et le haricot, la pomme de terre et autres légumes proviennent principalement des régions de l'Ouest et du Nord-Ouest du Cameroun, mais également de l'Adamaoua. Les tubercules, notamment le macabo, le manioc, la patate et la banane plantain proviennent principalement des régions du Sud, du Centre, du Littoral et de l'Est du pays. Les régions de l'Adamaoua, du Nord et de l'extrême Nord fournissent principalement des produits tels que l'oignon, l'ail et l'arachide.

En termes de volume, la figure 2 représente une connexion entre le marché de Kyé-Ossi et un hinterland qui recoupe l'ensemble du territoire camerounais.

${ }^{47}$ Non communément attribué aux transporteurs clandestins. 
Figure 2 : Les bassins d'approvisionnement du marché de vivres de Kyé-Ossi

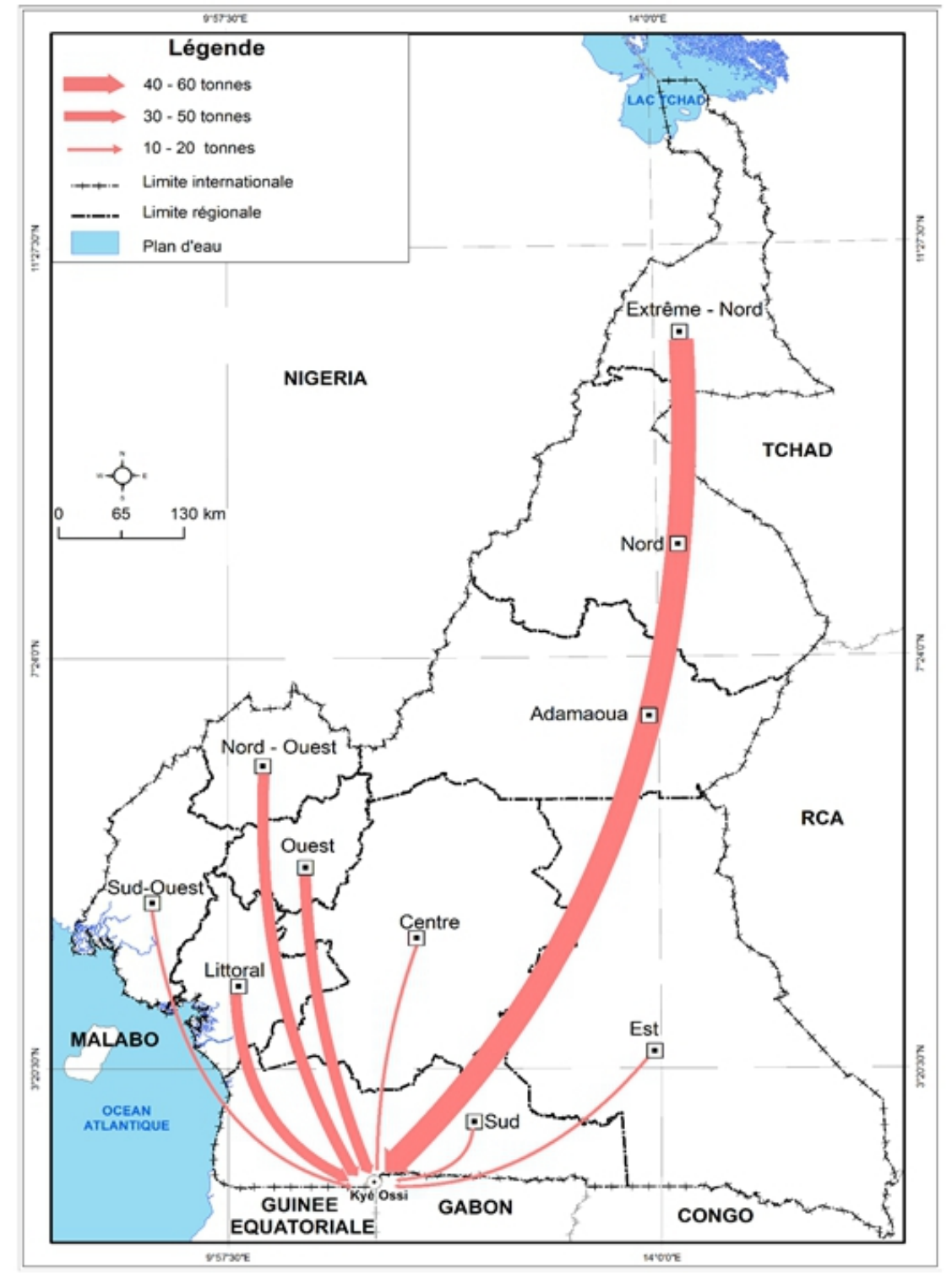

(Source : Carte administrative du Cameroun, enquêtes de terrain ; Conception et réalisation : Tchinda, 2018)

En plus des bassins de production ci-dessus cités, la figure 2 montre que la région du Sud produit également des spéculations à destination du marché de Kyé-Ossi, malgré un flux relativement réduit. Aussi, il est important de remarquer la présence de la Région du Sud parmi les bassins de production. On note donc deux types d'hinterland : un hinterland proche, 
constitué des bassins de production périphériques au marché de Kyé-Ossi, et un hinterland lointain, avec le reste des régions du Cameroun. Avec cette interconnexion avec les bassins agricoles dans l'arrière-pays camerounais, ce marché a pu développer un circuit de commercialisation des produits agricoles au profit de la Guinée Équatoriale et du Gabon.

\section{De Kyé-Ossi vers l'étranger : Un circuit dense de commercialisation au profit de la Guinée Équatoriale et du Gabon} Une demande spécialisée selon le pays

Le marché de Kyé-Ossi est un important point de distribution des produits agricoles exportés par voies terrestres vers la Guinée Équatoriale et le Gabon. Le tableau 1 ci-après indique une estimation des quantités, mais aussi les qualités des produits sollicités sur ce marché par les acheteurs gabonais et équato-guinéens.

Tableau 1 : Produits écoulés sur le marché de Kyé-Ossi par semaine

\begin{tabular}{|c|c|c|}
\hline Produits & $\begin{array}{c}\text { Quantité (tonne } \\
\text { par semaine) }\end{array}$ & Destinations \\
\hline Banane plantain & 84 & Gabon*, Guinée Équatoriale \\
\hline $\begin{array}{c}\text { Ananas, pastèque, banane } \\
\text { douce, papaye, avocat }\end{array}$ & 02 & Gabon, Guinée Équatoriale* \\
\hline Carotte & 04,5 & Gabon, Guinée Équatoriale* \\
\hline Poivron & 02,4 & Gabon, Guinée Équatoriale* \\
\hline Oignon & 55 & Gabon, Guinée Équatoriale \\
\hline Pomme de terre & 19 & Gabon*, Guinée Équatoriale \\
\hline «âton de manioc» & 08 & Gabon, Guinée Équatoriale* \\
\hline Maïs & 03,5 & Gabon*, Guinée Équatoriale \\
\hline Haricot & 04 & Gabon*, Guinée Équatoriale \\
\hline Source
\end{tabular}

Source : Enquête de terrain, 2018

*Principaux acheteurs

Il ressort du tableau 1 qu'il existe une certaine spécialisation dans l'approvisionnement des produits sur le marché de Kyé-Ossi. Les commerçants Gabonais sont plus portés vers les tubercules et les céréales tandis que les Équato-guinéens achètent plus des fruits, les légumes et les «bâtons de manioc ». Ceci peut s'expliquer autant par les habitudes alimentaires que par la distance qui sépare les villes où sont écoulés ces produits (figure 3). Les fruits et légumes pourrissent en effet très rapidement. Par conséquent, ils sont achetés par les commerçants qui opèrent dans les marchés proches de la frontière camerounaise tandis que les tubercules et les «bâtons de manioc », relativement moins périssables, sont sollicités par les vendeurs qui interviennent dans les marchés de l'arrière-pays du Gabon (Libreville, Franceville). 
Figure 3 : Représentation cartographique des quantités de produits vivriers du marché de Kyé-Ossi exportées vers les différentes villes des pays voisins

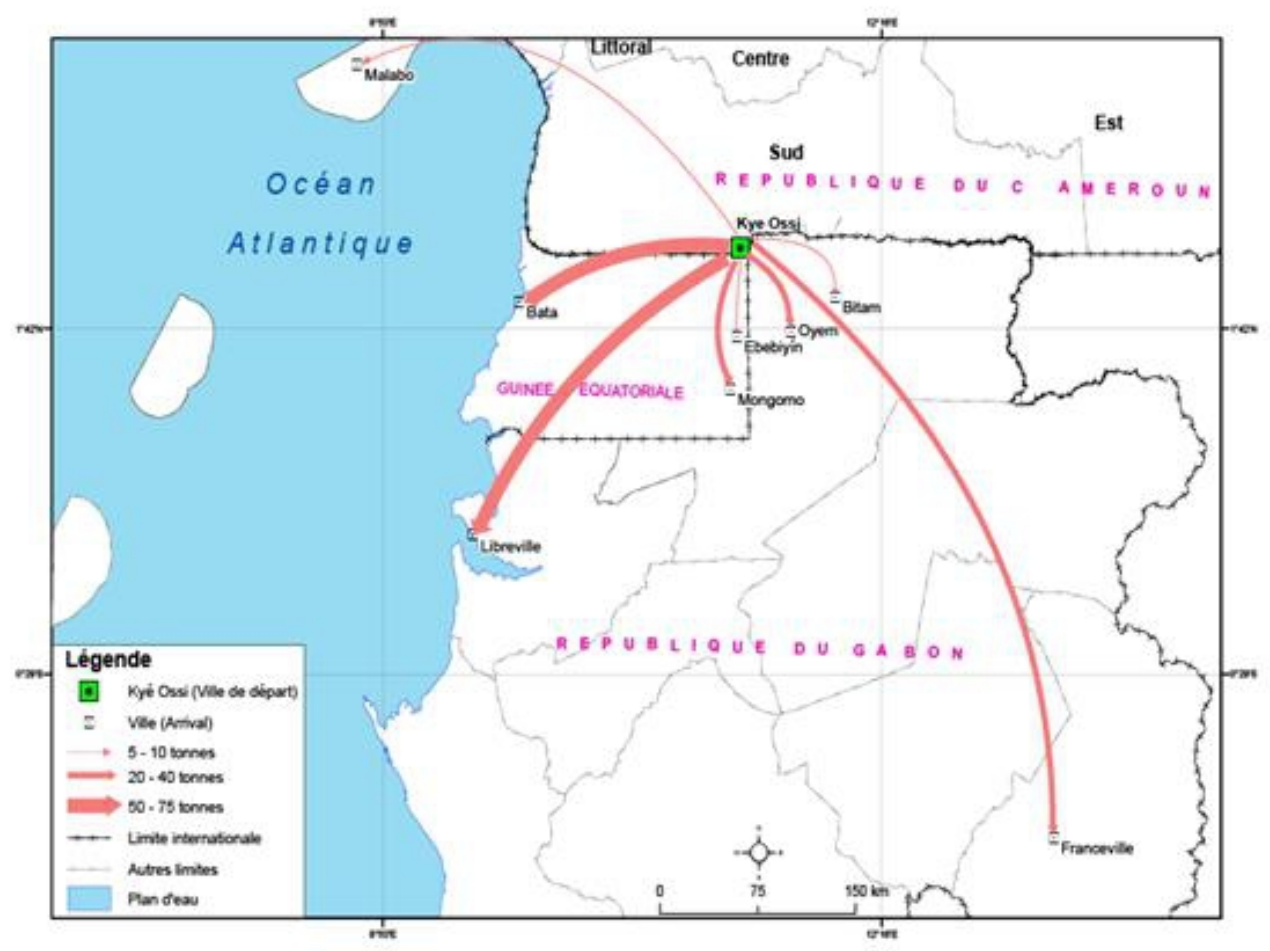

(Source : Enquête de terrain, conception et réalisation : Tchinda, 2018)

La figure 3 indique que les villes de Bata et Libreville concentrent plus 50 à 75 tonnes de produits par semaine, suivis des villes de Oyem, Mongomo et Franceville (20-40 tonnes). Les villes de Bitam et Ebebeyin concentrent moins 10 tonnes par semaine. Cette répartition très inégale des destinations des produits est plus liée au poids démographique des villes, et par conséquent à l'importance du marché.

\section{Mobilités entre le marché de Kyé-Ossi et les pays voisins}

De par la place centrale que le marché de Kyé-Ossi occupe dans la distribution des produits agricoles, il génère un ensemble de flux qui entretient ou facilite la mobilité des personnes et des biens dans cet espace frontalier. D'ailleurs, 35\% des commerçants qui travaillent dans le ce marché sont recrutées parmi les personnes ayant échouées à une ou plusieurs tentatives d'immigration vers le Gabon ou la Guinée Équatoriale. Ainsi, outre les avantages financiers que cette activité leur procure, elle leur sert de tremplin pour tisser de nouveaux contacts et entreprendre un nouveau « voyage ».

Les «bayam sellam» et les commerçants Gabonais et Équatoguinéens sont les acteurs les plus mobiles parmi tous ceux qui investissent ce 
marché. Les premiers effectuent deux fois par semaine des navettes entre les bassins de production et le marché. Leurs séjours dans les bassins de productions durent généralement entre deux jours et une semaine. Les déplacements, spécifiques selon le type de produits sont pour l'ensemble portés vers les régions de l'ouest, du centre et de dans les campanes du sud. Ils ne disposent pas pour l'immense majorité d'un moyen de transport personnel. Ils ont donc recours aux transports en commun (agences de voyage et clandos ou «Opep ») qui desservent Kyé-Ossi et Yaoundé tous les jours. Une fois par semaine, précisément le jeudi, des cars de transport relient KyéOssi à Bafoussam dans la région de l'ouest et Douala dans le littoral. Une fois la marchandise collectée dans les bassins d'approvisionnement, les services des camionneurs spécialisés sur la ligne sont sollicités pour l'acheminement des produits vers le marché.

La présence des commerçants gabonais et équato-guinéens se justifie justifient par la proximité (10\% des enquêtés), la qualité des produits commercialisés (39\% des enquêtés) et le niveau des prix pratiqués $(51 \%$ des enquêtés). Ils se regroupent généralement en petits groupes en fonction de leur marché d'attache et investissent le marché les lundis, mercredis et vendredis. Leurs mobilités sont dans bien de cas quotidiennes et leurs présences sur le marché varient entre douze et vingt-quatre heures. Il convient néanmoins de noter que les commerçants les plus proches (moins de $5 \mathrm{~km}$ ) du marché s'y rendent quotidiennement. Il s'agit dans la plupart des cas des détaillants qui ne disposent pas d'assez de revenus pour constituer d'importants stocks de marchandises. Ces derniers sont recrutés aussi bien parmi les locaux que parmi les étrangers en occurrence les équato-guinéens. Tous comme les «bayam sellam », les détaillants font recours aux services des transports en commun et des camionneurs pour se porter aisément sur le marché de Kyé-Ossi.

C'est dire que les transporteurs se positionnent comme un maillon essentiel de l'échange sur ce marché frontalier. Ils sont sollicités tant par les «bayam sellam» pour aller collecter les produits dans l'hinterland camerounais que par les acheteurs Gabonais et Équato-guinéens pour aller livrer les marchandises dans les différents points de chute. Les moyens utilisés pour ces mobilités sont fonction de la distance par rapport au marché et de la quantité de marchandises à transporter. La figure 4 ci-après renseigne sur la densité de ces déplacements. 
Figure 4 : Nombre de voyages effectués par semaine par les transporteurs du marché de vivres de Kyé-Ossi pour les pays voisins

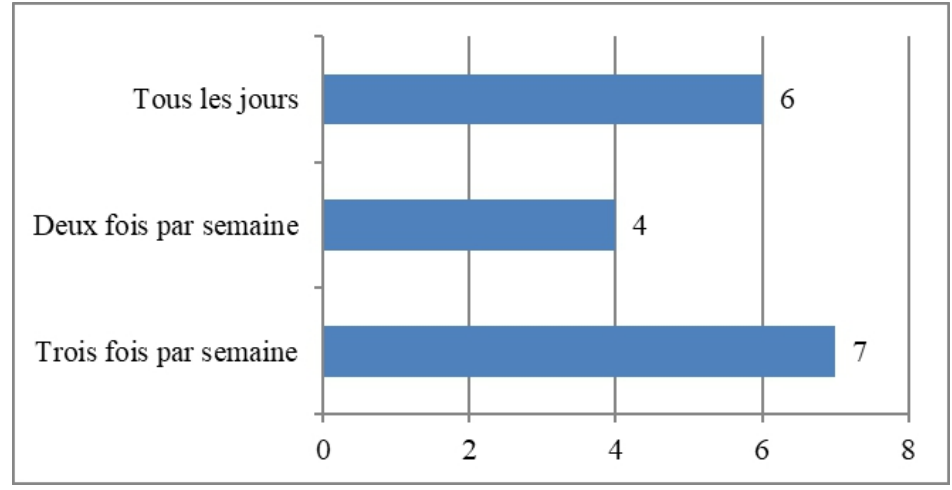

(Source : Enquête de terrain, 2018).

Il apparait à la lecture de la figure 4 que la plupart des transporteurs enquêtés font au moins trois (03) voyages par semaine, soit un voyage tous les jours d'intenses activités du marché. Les commerçants les plus proches du marché et les détaillants utilisent pour la circonstance des pousse-pousse, des taxis de brousse, des véhicules à usage personnel pour ceux qui en ont, et des cars de transport en commun pour se ravitailler (planche 2).

Planche 2 : Aperçu des moyens de transport utilisé par les petits acheteurs non loin de la frontière

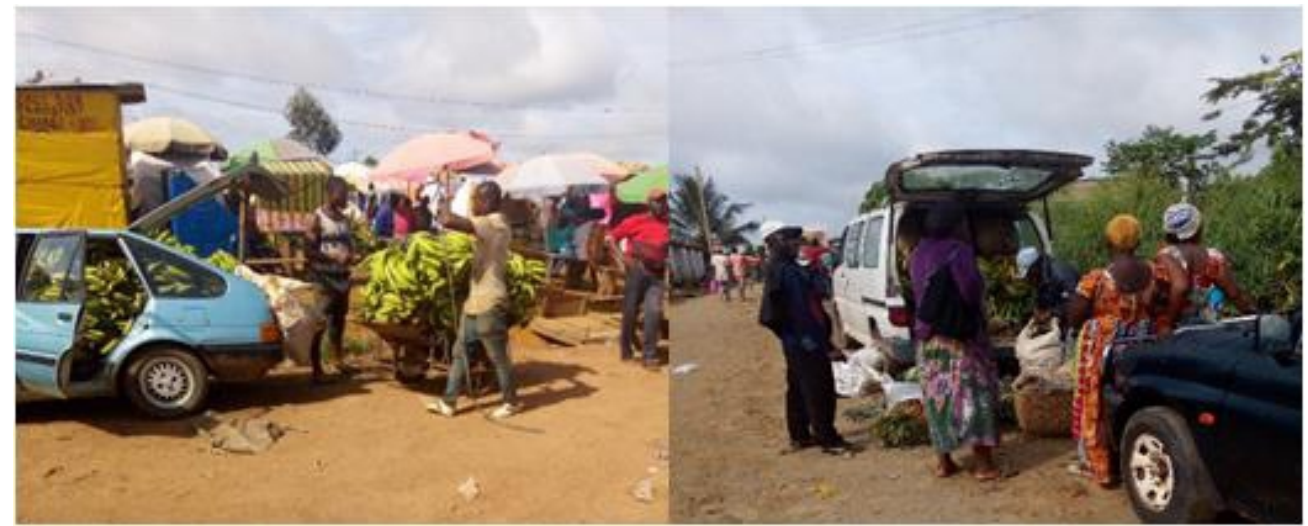

(Source : Image d'enquête, 2018)

Ce système d'échange, bien que très organisé fait face cependant à de multiples contraintes.

\subsection{Le marché de Kyé-Ossi : espace d'échange aux contraintes multiples}

Les acteurs qui interviennent dans le marché de Kyé-Ossi sont confrontés à de nombreuses difficultés (figure 4). 
Figure 5 : Problèmes rencontrés par agent de l'échange sur le marché de Kyé-Ossi

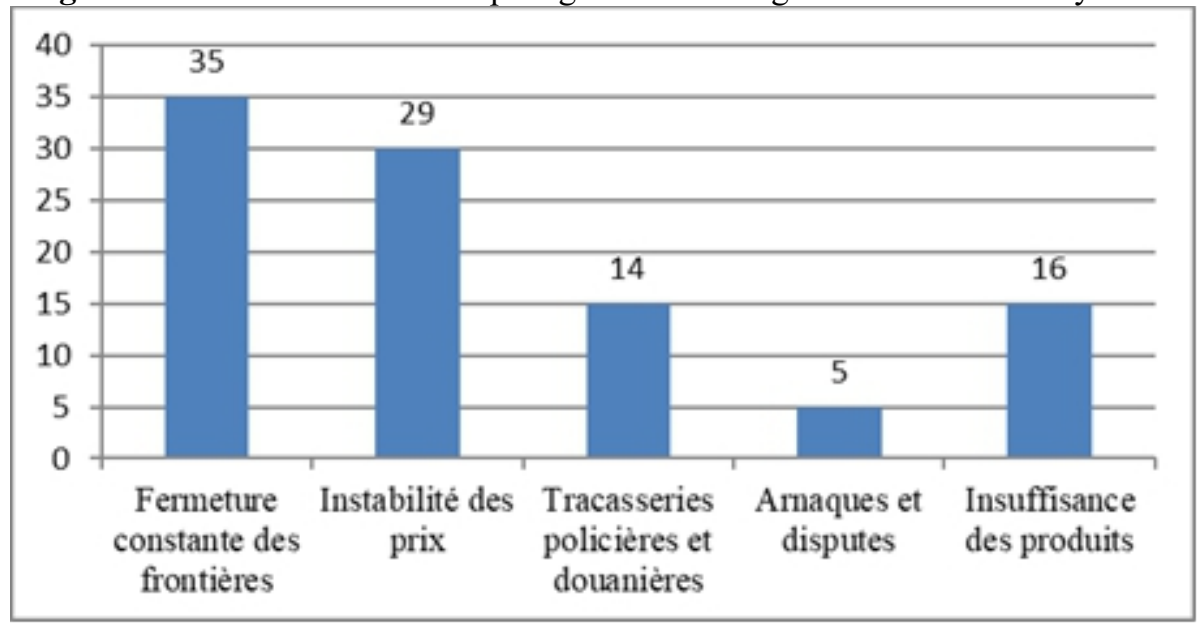

(Source : Enquêtes de terrain, 2018)

La figure 5 montre que les problèmes identifiés par les principaux acteurs sont, par ordre d'importance, la fermeture constante des frontières (35\%), l'instabilité des prix $(29 \%)$, les tracasseries policières et douanières (14\%), l'insuffisance des produits (16\%) et l'arnaque des commerçants (5\%). La fermeture des frontières se présente comme le problème le plus marquant, qui contraste cependant avec la volonté des Chefs d'État de la Sous-région à promouvoir le libre-échange. L'exemple le plus illustratif est la fermeture de la frontière Équato-guinéenne avec le Cameroun décidée par la Guinée Équatoriale entre décembre 2017 et janvier 2019. Cette décision avait été motivée par des raisons de sécurité. Au cours de cette période, les échanges entre ces deux pays n'étaient possibles que les mardis et vendredi et seuls les Équato-guinéens étaient autorisés à se porter au-delà de leur frontière. Le ralentissement de l'activité commerciale dans la ville en raison de cette fermeture a alimenté plusieurs marches de protestation des commerçants de Kyé-Ossi qui s'indignaient du manque de promptitude des autorités camerounaises dans les négociations avec leurs homologues Équato-guinéens pour la réouverture des frontières. Aussi, on note que malgré les accords de libre-échange entre le Cameroun et les autres pays de la Sous-région, c'est le Gabon et la Guinée Équatoriale qui proposent et animent les mécanismes d'échange entre ces pays, du fait que près de $80 \%$ des clients du marché de Kyé-Ossi viennent de ces deux pays. Ce qui confirme un malaise profond, relevé par l'Union Africaine (2019:7) à savoir que «malgré le riche potentiel inexploité de ressources de la région CEEAC, le potentiel intra-CEEAC reste très faible. Une infrastructure médiocre, des procédures douanières et d'immigration restrictives, des conflits persistants, une mauvaise coordination 
des politiques ainsi que d'énormes contraintes financières et humaines sont quelques-uns des défis qui doivent être relevés ».

Alors que la question de la fermeture des frontières par les pays voisins du Cameroun est présentée côté Cameroun comme le principal problème à la bonne marche des échanges transfrontaliers, les grossistes gabonais ou équatoguinéens se plaignent très souvent de l'instabilité des prix et de l'arnaque des commerçants camerounais qui sont finalement, en l'absence d'un véritable cadre règlementaire pour organiser ce marché fait des grossistes les maitres du jeu.

\section{Discussion}

Le marché de Kyé-Ossi constitue un lieu de concentration et un nœud important dans les réseaux d'approvisionnement et de ravitaillement en produits vivriers entre le Cameroun, le Gabon et la Guinée Équatoriale. Il favorise les échanges entre ces trois pays à partir des flux de mobilité qu'il crée et entretient. Cependant, ces mobilités se concentrent essentiellement autour du marché ou tout au plus à quelques auberges et bars avoisinants. Toute chose qui offre moins de perspectives à la structuration d'un système de birésidentialité ou de multilocalité transfrontalière qui favoriserait une intégration sociale sous régionale à partir de ce marché. Bien plus, ces mobilités qui se font essentiellement à la « carte »sont davantage pratiquées par les grossistes, les transporteurs et ménagères environnantes qui ont une certaine liberté de mouvement. Cependant, les autres populations, principalement les Camerounais peinent à commercer au-delà de leur frontière sans remplir des formalités administratives. Pourtant, on se serait attendu à ce que la densification des réseaux commerciaux transfrontaliers autour du marché érode progressivement les prérogatives étatiques et facilite dans une certaine mesure la fin des frontières tel que le relève Bennafla (1999, p.37). On est ainsi loin d'une dynamique d'intégration par le « bas » à partir des réseaux marchands qui, comme le souligne Grégoire (2002, p.139) modifient les organisations territoriales.

Le temps de séjour des commerçants Gabonais et Équato-guinéen en territoire camerounais est relativement bref. Cette breveté des séjours des agents de l'échange associée à leur faible représentativité sur le marché, leur offre moins de possibilités de constituer des communautés d'affaires pouvant leur permettre d'infléchir les coûts et de réduire les incertitudes du marché comme c'est le cas sur les marchés frontaliers de Gaya-Malanville-Kamba et Birni N'konni en Afrique de l'Ouest avec les commerçants Nigérians (Mahaman Moustapha, 2015, p129-149). L'on se retrouve dès lors dans un marché compartimenté où les agents obéissent à des spécialisations particulières dans les circuits d'achat, de vente et même dans les jours de fréquentation. 


\section{Conclusion}

La présente réflexion s'est inspirée du rôle central que le marché frontalier de Kyé-Ossi joue dans l'approvisionnement des pays voisins en produits vivriers. En effet, ce marché se positionne comme une plateforme qui concentre les flux des produits vivriers pour les redistribuer vers le Gabon et la Guinée Équatoriale. En agissant de la sorte, il se positionne comme un tremplin à l'intégration sous régional et pose les ferments de la libre circulation tant souhaitée dans la zone CEMAC. Les échanges sont cependant très renfermés sous le carcan de la nationalité et empêchent le marché de développer son plein potentiel. En effet, dans sa configuration actuelle, les Camerounais, dans leurs postures de commerçants, ne sont pas autorisés à se porter au-delà de la frontière du Cameroun pour écouler leurs produits au Gabon ou en Guinée Équatoriale. Les Gabonais et les Équato-guinéens, principaux acheteurs n'ont pas le droit de se porter au-delà des limites du marché pour s'approvisionner. Cette situation inscrit les échanges dans un système imparfait où chacun utilise autant que faire se peut le monopole qu'il dispose pour faire fluctuer les prix. L'on peut donc comprendre l'appel lancé par les uns et les autres pour une ouverture effective des frontières.

\section{References:}

1. Ayiwoue Mbouemboue Edouard, Azeufouet Alain., Medjou Séraphine., Parrot Laurent, Temple Ludovic, Mars 2006. Quantification des flux transfrontaliers. Étude des flux transfrontaliers de produits agricoles et horticoles sur les frontières sud du Cameroun : phase I, rapport final Montpellier : CIRAD, 83 p.

2. Degavre Florence et Lemaître Andreia, 2008. « Approches substantives de l'économie : des outils pour l'étude des organisations d'économie sociale », Revue Interventions économiques [En ligne], 38 | 2008, mis en ligne le 01 décembre 2008, consulté le 25 mai 2019. URL : http://journals.openedition.org/ interventionseconomiques/313 ; DOI : 10.4000/interventionseconomiques.313

3. Dieye Cheikh Tidiane, 2006. «L'Afrique et le chevauchement des accords régionaux », Revue Interventions économiques [En ligne], 55 |, mis en ligne le 29 juin 2016, consulté le 14 juin 2019. URL : http://journals.openedition.org/interventionseconomiques/2815 ; DOI : 10.4000/interventionseconomiques.2815

4. Document de Stratégie pour la Croissance et l'Emploi (DSCE). 2009. Cadre de référence pour l'action gouvernementale pour la période 2010-2020, 174 p.

5. Grégoire, E., 2002. Territoires marchands en Afrique subsaharienne, dans Bart F. (coord.), Bonvallot Jacques (coord.), Pourtier R. 
(coord.) Regards sur l'Afrique, Historiens et Géographie n³79, pp 133-140

6. Ihle Rico, Amikuzuno Joseph et Cramon-Taubadel Stephan von, 2010. «L'intégration des marchés avec et sans échanges commerciaux directs : le cas de la tomate au Ghana », Revue d'économie du développement, De Boeck Supérieur, Vol. 18 |pp21- 46

7. Karine Bennafla, 1999. La fin des territoires nationaux ? État et commerce frontalier en Afrique centrale, dans politique africaine $\mathrm{n}^{\circ} 73$, pp 25-49

8. Mabaya Edward, 2004. «L'intégration spatiale de marchés et échange discontinu : une analyse des marchés spot urbains pour la tomate au Zimbabwe. », Économie rurale. N²83-284, pp. 62-71.

9. Mahaman Moustapha Mamadou Koné, 2015. Marchands ouestafricains et marchés frontaliers : construction d'une communauté d'affaires. Géographie. Université Michel de Montaigne - Bordeaux III; Université de Niamey. Français. ffNNT : 2015BOR30075ff. fftel$01399293 f$

10. Medjou Séraphine., Temple Ludovic., Azeufouet Alain., David Olivier., Parrot Laurant., Tsague Edouard., Ayiwoue Mbouemboue Edouard, 2007. «Coordinations des opérateurs et intégration économique en Afrique Centrale : caractérisation du commerce transfrontalier des produits vivriers et horticoles au Cameroun ». Communication à la conférence sous-régionale Mondialisation et réduction de la pauvreté en Afrique centrale. Groupe de Recherche en Économie Théorique et Appliquée (GRETA). Faculté des sciences économiques et de gestion appliquée (FSEGA). Université de Douala, Cameroun, $17 \mathrm{p}$.

11. Meyer A., 2006. L'intégration régionale et son influence sur la structure, la sécurité et la stabilité d'États faibles : L'exemple de quatre États centrafricains, Thèse de Doctorat de Science politique, Institut d'Études Politiques de Paris, 543p.

12. Plan Communal de Développement (PCD), 2014. Élaboration du Plan Communal de Développement, diagnostic participatif. Document 1 : Diagnostic institutionnel communal, $103 \mathrm{p}$.

13. Union Africaine, 2019. Rapport sur l'état de l'intégration régionale en Afrique. Résumé analytique, https://au.int/sites/default/files/newsevents/workingdocuments/35970 -wd-2.2. new_resume_executif_-_rapport_sur_letat_de_lintegrationfr.pdf, Consulté le 5 août 2019. 